$\mathbb{T}$ periodica polytechnica

\author{
Transportation Engineering \\ $37 / 1-2(2009) 23,28$ \\ doi: 10.3311/pp.tr.2009-1-2.04 \\ web: http://www.pp.bme.hu/tr \\ (c) Periodica Polytechnica 2009
}

RESEARCH ARTICLE

\section{Co-operative Transport System and investigation of the co-operation}

\author{
Gábor Szúcs
}

Received 2008-10-03

\begin{abstract}
This paper deals with Intelligent Transport Systems (ITS) and the concept of a Co-operative Transport System (CTS), in which the communication and the knowledge sharing is much more important. This distributed knowledge allows improving road transport operations more safety and efficiency. After showing some issues about development and advantages of a CTS, the communication part is discussed among CTS elements. The most important parts and usefulness of CTSs are shown with some examples, e.g. TRACKSS, which is based on the knowledge sharing sensors on the road network.
\end{abstract}

\section{Keywords}

Intelligent Transport System · esafety · information and communication technologies · Co-operative Transport System

\section{Acknowledgement}

The author would like to thank the leader of the TRACKSS project, Antonio Marqués for the possibility of the working in an EU project. Special thanks are due to Dr. J. Berényi and Prof. A. Jávor for leading the Hungarian participant of the project. Special thanks are due to Dr. J. Berényi and Prof. A. Jávor for leading the Hungarian participant of the project. This work was partly supported by the János Bólyai Research Scholarship of the Hungarian Academy of Science.

\section{Gábor Szücs}

Department of Telecommunications and Media Informatics, BME, Magyar Tudósok krt. 2, H-1117 Budapest, Hungary

e-mail: szucs@tmit.bme.hu

\section{Motivation}

A very high number of persons are killed, injured or disabled annually in road traffic crashes significant material damages are in parallel the consequences. Causes of the accidents may vary, but they can be categorized following different aspects: the road infrastructure, the vehicle, the driver and their interactions are covering the major groups of these causes. It is important to find bottlenecks where the potential of danger is high and where this risk should be communicated to the driver more directly more effectively - than only the traditional signalizations.

\section{Safety on roads and transport systems}

\section{1 eSafety}

The objective of the eSafety initiative is to accelerate the development, deployment and use of new technologies for increasing road safety in Europe. It was launched at the eSafety highlevel meeting on 25 April 2002 and its objective is $80 \%$ of the EU population to be served by 2010.

Recommendations will be formulated for further development of communication devices, to enhance safety, following the targets of the eSafety forum. New technology requirements will be defined e.g. how to build and implement self-configuring communication networks, including their reaction to an automatically created deviation plan for an obstacle arising from an accident.

The provision of information to influence the traffic flow management in case of accident can be used for more simple tasks. The access management based on this information may be used for optimising the long route planning - the communication tools, ways and the knowledge will be in part already available. eSafety (with a new service: eCall) may give new impetus for safe lives on the roads. eCall (communication possibility in the vehicle) may solve a series of communication problems actually not to be seen because of the non existence of co-operation between the infrastructure and the vehicle.

\subsection{Intelligent Transport System}

Intelligent Transport Systems frequently span several transport means (e.g. private and public transport) and are provided 
in many locations (on board vehicles, at the roadside or through mobile devices). The use of an ITS architecture also makes it possible to highlight any problems that arise from the refinement and modification of the services that are being provided. The architecture can become a "tool" that enables the traffic and transport problems to be addressed and resolved.

The first version of the European ITS Framework Architecture was published in September 2000. The content was developed by means of constant consultation on intermediate results with European Stakeholders and a panel of Wise-men. The final outcome was agreed and endorsed by the major European actors as the Framework Architecture to be used as the reference providing:

- The ability of travellers and freight operators to plan and implement journeys using the most efficient, convenient and cost-effective combinations of transport modes;

- Compatibility and consistency of information delivered to end-users through different media (any end-user should be able to receive the same information through different media such as GSM, etc.);

- Compatibility of equipment with different infrastructures, thus enabling "seamless" travel across Europe (e.g. a driver [11] should be able to use the in-vehicle system for electronic payment both at home and abroad);

- A basis for regional, national or European authorities to produce master plans and recommendations to facilitate ITS deployment allowing, for instance, authorities to co-operate on traffic management across borders and boundaries;

- An open market for services and equipment where compatible sub-systems are offered (no more ad-hoc solutions);

- Economies of scale in equipment manufacture permitting competitive prices and cheaper investments with compatibility guaranteed;

- A known market place into which producers can supply products with reduced financial risk.

Within Europe, new applications of ITS [12] are continually being developed by improved informatics. The informatics can help in the problem solving in the transportation as well [18]. It is crucial to ensure that their deployment is co-ordinated in a way that ensures the overall and long term improvement of transport efficiency. Without a common European ITS Framework Architecture, there is a risk that products providing ITS services will be developed in isolation and therefore not able to interact easily with each other. It may also become impossible for a consistent level of service to be uniformly provided to citizens throughout Europe. It is therefore essential for all parties involved in the implementation of ITS across Europe to adhere to an agreed common Framework Architecture. Such a Framework architecture must accommodate national plans and support efforts in research, standardisation, deployment and investment. It must ensure that new systems are compatible, but also include 'migration' plans for the incorporation and adaptation of existing or 'legacy' installations.

\subsection{Co-operative Transport Systems}

The problem of safety in transport [1] is especially acute in the case of road networks. On the other hand there are many safety standards in Europe and all over the world. Existing infrastructure will need to be operated up to (and sometimes beyond) its design margins in order to cope with the additional capacity. However, while doing so, safety standards must not only be maintained at current levels, but increased. The safety is based on autonomous or stand-alone systems, and there is no possibility to reach a high level safety without cooperation among the stand-alone systems, so the improvement is limited. In order to overcome the visible limitations of those systems, there is a need for another class of solution: Co-operative Transport Systems (CTS), whose intelligence is distributed/shared between road infrastructure and vehicles, compromising innovative approaches on transport safety and efficiency. Undoubtedly, today available (or under development) technologies enable the improvement of not only sensing systems, but also the way these systems share information. Smarter systems demand smarter sensors; cooperative systems require cooperative sensors. The above mentioned idea can be improved and developed a number of breakthrough sensing technologies and a new model for knowledge sharing between sensors.

In this context, stand-alone Intelligent Transport Systems (ITS) are not any longer the solution. Instead, Co-operative Transport Systems (CTS) are the most promising option. In this paradigm, analogous to that of the Ambient Intelligence, the different actors involved in ITS - humans and machines - will interact among each other in order to meet their individual goals while at the same time maximizing the safety and efficiency of the overall system. Systems which can deliver smarter functionalities are needed. However, in order to achieve these smarter systems, better input data is required as well.

Summarizing the above mentioned aims the objectives are the following: contribute towards improving road safety, optimise the efficiency of the road network, improve the road transport environment, establish new advanced sensing systems, and integrate all of them into CTS. It is also increasingly important that they should be fully compatible, not only within a single country, but at the international level as well.

\section{Operational framework}

\subsection{The sensors and the shared information}

The objective is to develop and/or radically improve a number of innovative sensing technologies including fixed on-theground, mobile on-the-ground, remote sensing and on-board vehicle sensors. The sensors are the following:

- Enhanced Laser-based Scanners 
- Inductive Super Loops

- Smart Video Cameras

- Advanced Ice Detectors

- High-dynamic High-resolution CMOS Camera

- Pedestrian Detectors

\section{- Radar Sensors}

- Weather station

The sensors are intended to be self-contained elements to be used as building blocks of other cooperative systems, being easy to add these new sensors to other existing architectures. In the following the details of the sensors are described.

All of the laser scanner systems use one of these two sensors. Two different systems are mainly used to detect vehicle speed: laser scanner perpendicular to the road and laser scanner multifunction. In the first case the system is based on two perpendicular laser beams focus to the road that are interrupted successively by the crossing of vehicles. The distance between these two laser beams is known and the vehicle speed can be obtained by this configuration. The values of vehicle speeds are shared among the other elements of CTS.

Several methods have been applied toward the general category of road surface condition sensing. The presently used technology for ice detection consists of a thermometer: it is clear that the information coming from the air temperature measurement only is very partial and can be considered just as a generic warning. The idea is the introduction of a new principle to detect the road surface conditions: no more just an air temperature measurement but a new method. The proposed approach consists of the IR (Infrared Range) spectral analysis of the road surface. The binary value of the measurement is very important information for other elements of CTS, particularly for the drivers in the vehicles.

Detecting real-time traffic conditions is a key element to advanced traffic management and traveller information systems. Until the last decade, inductive loop detectors were the conventional method for collecting permanent on-sites traffic data. In recent years, technological innovations have given rise to many new and different types of advanced traffic detectors using magnetic, ultrasonic, microwave, infrared, and video sensors (video detection system). They provide direct measurement for counting, occupancy measurement, presence detection, queue detection, speed estimation and vehicle classification, and they share the information in CTS.

Vehicles-behaviour analysis using video systems are usually based on CCTV cameras with a set-up optimised for normal traffic surveillance. Abnormal flow behaviour is recognised and after it the sensor provides an indication of the occurrence of an incident. Intersection control software provides accurate presence and queue detection in addition to vehicles counts. Moreover, video has now the real potential to be used in detecting pedestrians and cyclists.
There are some potential benefits of the combination between two complementary technologies. E.g. the combination of the AC20 radar and the video sensing system can lead to an enhanced sensing solution. The success of this cooperation will lay in the different sensing approach of sensors, and the complementary sensors capacity to fill the gaps of one another.

We should focus on automatic exchange of information between the infrastructure and vehicles. Vehicles, as mobile elements, will be used to collect data about weather and traffic conditions, this information will be combined with data observed by the corresponding vehicle. This aggregated information (e.g. an event in a time, in a given place measured by rear, front, lateral distance, etc.) will be used to control traffic signals, inform drivers automatically and give advice about the optimal route.

\subsection{Communications infrastructure}

The elements of the co-operation among operators, infrastructure, vehicles, their drivers and other road users will be established and supported with the aim to deliver more efficient, safe, secure and comfortable journey - as summarised the goals of the Co-operative Transport Systems (CTS). The essence of the co-operative systems is that they involve two-way communications, with interactions real-time. The vehicle may communicate directly with one another, so that a driver knows for example if another vehicle is on a conflict course or, vehicles may provide data directly to traffic management and receive individual guidance and in support return. Essentially, these systems can greatly extend the driver's range of perception, providing relevant information on the behaviour of traffic in general and neighbouring vehicles in particular beyond the range of vision.

The generic concepts should share information by the following ways:

- Procedures to convert different units - information must be coherent.

- The information should be accurate [10].

- Communication processes between sensors - the different devices must be identifiable.

- Communication processes between sensors and CTS - a generic and standard solution is intended.

- The knowledge should be capable for data integration techniques such as Bayesian inference [13, 17], Dempster-Shafer inference, Fuzzy Logic [14], and Artificial Neural Networks [13].

The research is based on the existing and emerging communications technologies in order to use the best way for knowledge sharing among all the objects included in the system. The system will be open, interoperable and scalable allowing easy upgrading. It requires an Operational Framework to make possible the co-operative experience, to guarantee a set of basic functionalities, that are indispensable when making sensors share their 

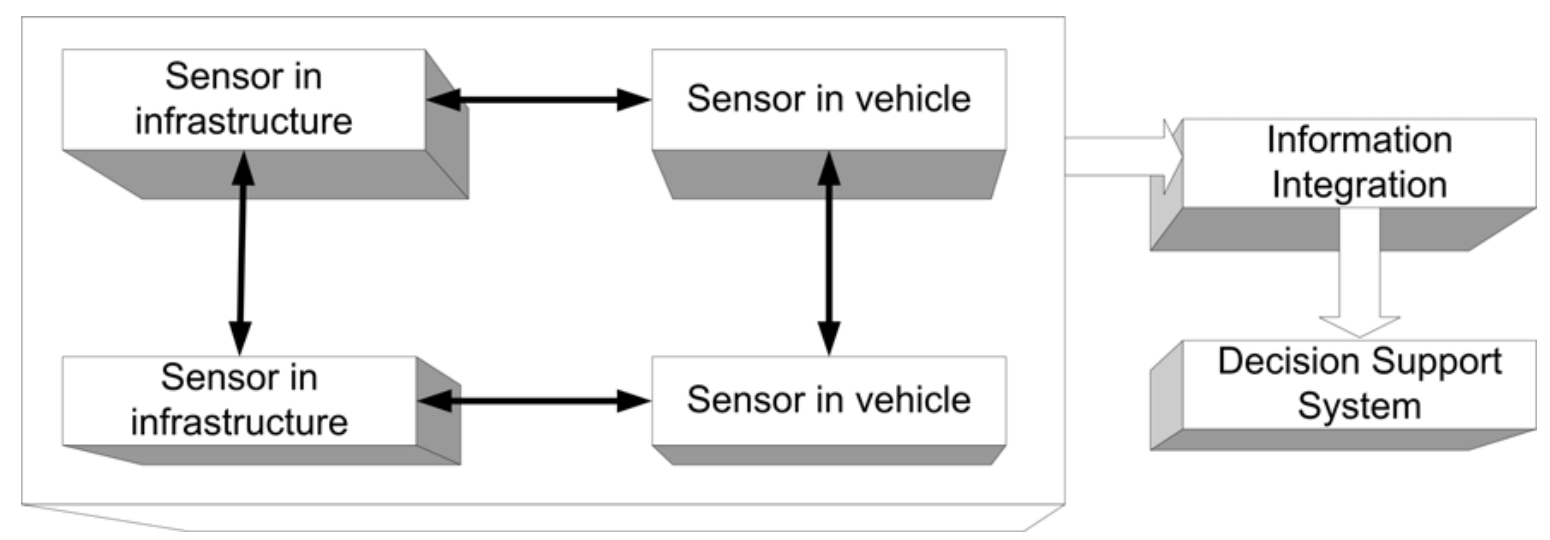

Fig. 1. Structure of the Operational Framework

knowledge. The Operational Framework defines these different functionalities and how they are related to each other (see Fig. 1). The core of the architecture should accomplish the following functions:

- To provide a mechanism to manage the exchange of information among all the components of the system (particularly among sensors).

- To monitor the physical status of the different devices constituting the sensors infrastructure. In case of failure - due to a link problem or a sensor in a bad state - the architecture should detect the problem and propose solutions, if possible.

- To provide a fault-tolerant communications infrastructure. This system will not propose new innovative telecommunication solutions, but will tackle inevitably the development or implementation of specific communication systems. Information and Communication Technology (ICT) in industry is highly developed, so the best communications technology should be found for knowledge sharing between all the system elements.

\subsection{Communication technologies for fixed elements}

Communication technologies enabling the interconnection of fixed elements could be

- Wired (xDSL, Cable) or

- Wireless (WLAN WIFI for extended area coverage, Bridge WIFI for point to point communications, DSRC or Bluetooth for shorter distances and road signs). A general model of wireless cellular network is already published (for example: [16]).

At planning a CTS system the distance between the elements are large and the key items are the vehicles, so in the following I depict the mainstream technologies for mobile elements, these possibilities are wireless technologies.

\subsection{Communication technologies for mobile elements}

Mobile network technologies have evolved from analogue based systems to digital based systems and from circuit switching to packet switching technologies. This evolution can be described by different generations of mobile technologies, i.e. first-generation $(1 \mathrm{G})$, second-generation $(2 \mathrm{G}), 2.5 \mathrm{G}$ and thirdgeneration $(3 \mathrm{G})$ technologies. Only $1 \mathrm{G}$ is based on analogue technology. Some of the main standards [8] for each generation technology are:

- 1G: Total Access Communication System (TACS) in Europe, Advance Mobile Phone System (AMPS) in North America, Nippon Telegraph \& Telephone (NTT) in Japan, Code Division Multiple Access One (CDMAONE).

- 2G: Global System for Mobile Communication (GSM), Code Division Multiple Access 2000 (CDMA2000), High Speed Circuit Switched Data Technology (HSCSD).

- 2.5G: General Packet Radio System (GPRS), Enhanced Data Rate for GSM Evolution (EDGE).

- 3G: Universal Mobile Telephone Standard (UMTS).

\subsection{Comparison of some communication standards}

Global System for Mobile Communication (GSM) is a second generation standard for mobile communication, developed by the European Telecommunications Standards Institute (ETSI) and now currently owned by the Third Generation Partnership Project (3GPP). From the point of view of the communication of ITS/CTS, the key advantage of GSM systems has been early delivery of new services at low costs. The advantage for network operators has been the low infrastructure cost which is caused by open competition. The primary disadvantage has been that GSM's radio network is based on TDMA technology, which is considered less advanced than competing CDMA-based systems [3]. The other disadvantage is the low performance.

General Packet Radio Service (GPRS) is packet switched wireless protocol providing non-voice value added services that allows information to be sent and received across a mobile telephone network. At the planning of a CTS the transit delays of 
Fig. 2. The solutions in Transport Systems before and after the TRACKSS
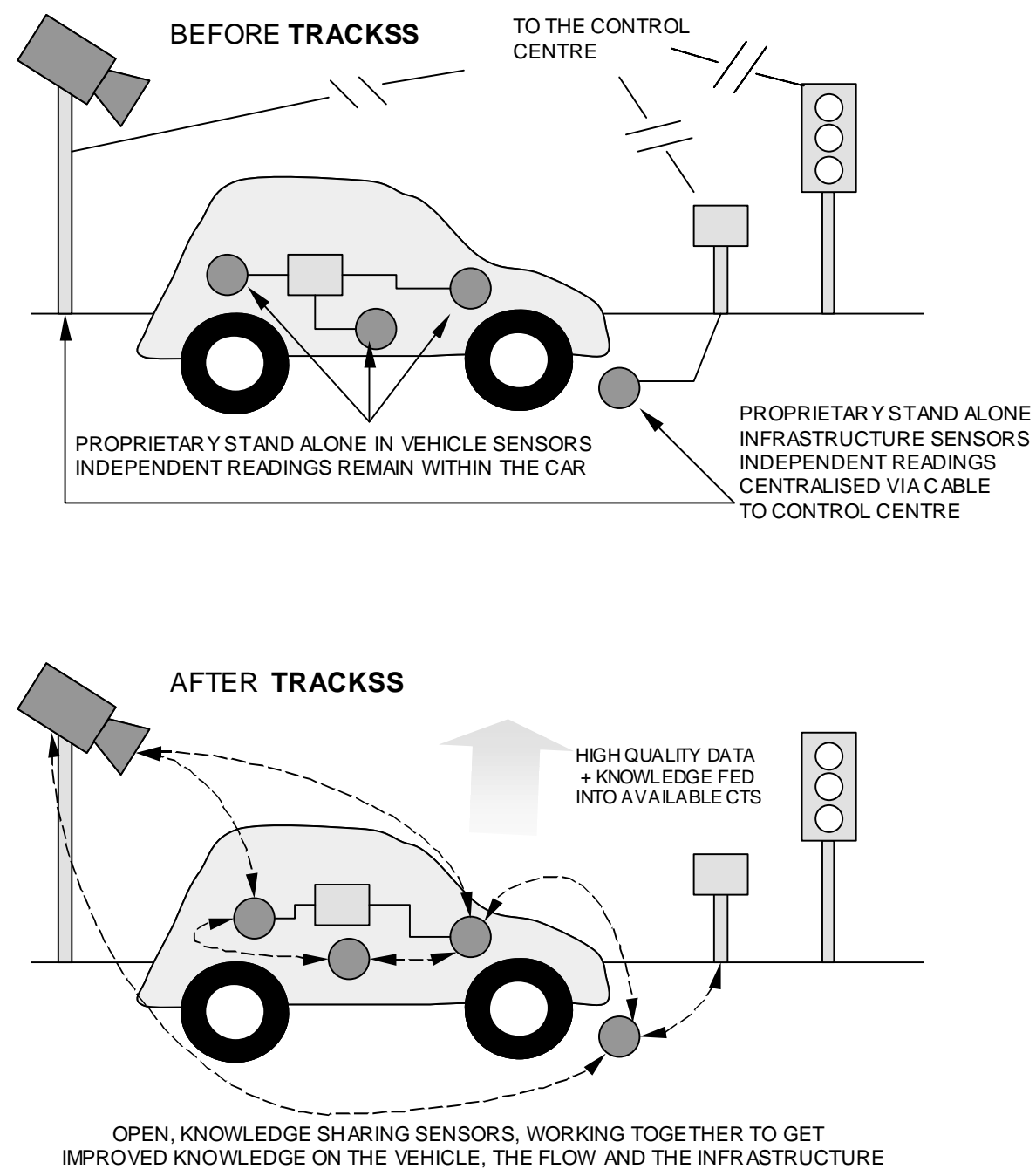

GPRS should be considered, that is data corruption or data loss may occur because GPRS sends data packets through different channels to reach a destination.

WIFI 802.11 p - WAVE (WAVE: Wireless Access for the Vehicle Environment). WAVE defines enhancements to 802.11 required to support Intelligent Transportation Systems (ITS) applications. This includes data exchange between high-speed vehicles and between these vehicles and the roadside infrastructure in the licensed ITS band of $5.9 \mathrm{GHz}$. It expands on conventional 802.11 wireless networking to allow for provisions that are specifically useful to automobiles. 802.11p is used as the groundwork for Dedicated Short Range Communications (DSRC), or automotive targeted communications that can occur both between vehicles and from a vehicle to a network of roadside infrastructure.

In the communication investigation the GSM, GPRS and WIFI $802.11 \mathrm{p}$ - WAVE communication standards are examined. GSM and GPRS protocols are compared in [2], the data trafficflow in the communication can be analyzed by theoretical [9] or by stochastic modelling [7].

\section{TRACKSS project dealing with cooperation}

The extended ITS systems lead to Cooperative Transport Systems (by extension with cooperative functions), in this part of the paper the most important projects dealing with cooperation are collected.

KAREN (Keystone Architecture Required for European Networks) project (1998-2000) [5, 6] is developed as the first version of the European ITS Framework Architecture. The FRAME projects (FRamework Architecture Made for Europe) [4] were funded by the European Commission as part of the 5th Framework Programme of the Information Society Technologies (IST) Directorate. FRAME-NET (July 2001-Sept 2004) was a 'Thematic Network' whose role was to provide information about the European ITS Architecture and promote opportunities for those involved in architecture-related activities in Europe to exchange their experience. THEMIS - Thematic Network [19] whose aim was to optimise management of Intermodal Transport Services. WATERMAN - Thematic Network which has the aim of reaching common understanding regarding concepts of VTMIS (Vessel Traffic Management and Information Systems) and of RIS (River Information Services). Close liaison with the FRAME projects with regard to intermodality [15.22]. VIKING - a project with the aim of co-ordinating the implementation of ITS in Northern Europe [21].

TRACKSS (Technologies for Road Advanced Cooperative Knowledge Sharing Sensors) is an EU project starting in 2006 
and finishing in 2009 [20].

Synchronised with the eSafety programme of the EU the wide approach - aiming at supporting the network planning and management, road traffic modelling, management, monitoring, control the project will have essential effects on more factors of the safety and hopefully on fatalities accidents statistics.

These were the global aims, and in order to reach this purpose the task is the development of a new class of solution: Cooperative Transport Systems (CTS), where intelligence is distributed among the vehicles and the road infrastructure. The TRACKSS will contribute to and seek compatibility with a panEuropean e-Safety architecture. The consortium developing this ambitious project counts with 15 partners, including global industrial players, innovative SMEs and world-class research institutions from Spain, UK, France, Germany, Italy, Hungary and the Czech Republic.

The objective of the CTS can be seen in the Fig. 2, where the situation before and after the project is shown.

\section{Summary}

This paper has shown the researching and developing innovative sensing technologies and cooperative functions that will provide a new approach for transport systems making them cooperative by the development of a brand new set of safety functions and new devices. Using cooperative sensors we will get more punctual and much more information about circumstance, which means large benefit at critical situations. The main point of our research is the development of trustful new technologies enabling a breakthrough in the current systems for sensing and predicting flow, infrastructure and environmental conditions surrounding traffic, with a view to improve road transport operations safety and efficiency.

Wireless network technologies are getting more and more widespread both in telecommunications and in computer networks. After an overview of current wireless technologies and their possible uses in Transport System environment, a specific spreading application is investigated: making a transfer through CTS. This research is the first step of an overall analysis of a developed CTS system.

\section{References}

1 Abdel-Aty M A, Pemmanaboina R, Calibrating a Real-Time Traffic CrashPrediction Model Using Archived Weather and ITS Traffic Data, IEEE Transactions on Intelligent Transportation Systems, June 2006, pp. 167-174, DOI 10.1109/TITS.2006.874710.

2 Bettstetter, GSM Phase 2+, General Packet Radio Service GPRS: Architecture, Protocols, and Air Interface, IEEE Communications Surveys, Vol. 2, 1999. Third Quarter.

3 Chung Y U, Cho D H, Enhanced Soft-Handoff Scheme for Real-Time Streaming Services in Intelligent Transportation Systems Based on CDMA, IEEE Transactions on Intelligent Transportation Systems, June 2006, pp. 147-155, DOI 10.1109/TITS.2006.874715.

4 FRAME projects, 2005, available at http://www. frame-online.net/

5 Franco G, Architecture Experiences in the Urban Environment, 2000, available at http://www. frame-online.net/Articles/EU-ITS2000.DOC
$6 \_$, Deployment of European ITS Framework Architecture, 2001, available at http://wWw. frame-online.net/Articles/IEEE-ITSC2001. DOC

7 Gyimesi Cs, Vezeték nélküli hálózatok vizsgálata szimuláció segítségével, Budapest, 2006. M.Sc. Thesis, BME.

8 ISO (International Organisation for Standardisation), available at http:// WWW.15o.org

9 Lencse G, Muka L, Convergence of the Key Algorithm of Traffic-Flow Analysis, Journal of Computing and Information Technology - CIT 14 ( 2006), no. 2, 133-140, DOI 10.2498/cit.2006.02.04.

10 Lo H K, A Reliability Framework for Traffic Signal Control, IEEE Transactions on Intelligent Transportation Systems, June 2006, pp. 250-260, DOI 10.1109/TITS.2006.874680.

11 McCall J C, Trivedi M M, Video-Based Lane Estimation and Tracking for Driver Assistance: Survey, System, and Evaluation, IEEE Transactions on Intelligent Transportation Systems, March 2006, pp. 20-37, DOI 10.1109/TITS.2006.869595.

12 Munaka T, Yamamoto T, Watanabe T, A Reliable Advanced-Join System for Data Multicasting in ITS Networks, IEEE Transactions on Intelligent Transportation Systems, December 2005, pp. 424-438, DOI 10.1109/TITS.2005.858623.

13 Oh C, Oh J S, Ritchie S G, Real-Time Hazardous Traffic Condition Warning System: Framework and Evaluation, IEEE Transactions on Intelligent Transportation Systems, September 2005, pp. 265-272, DOI 10.1109/TITS.2005.853693.

14 Quek C, Pasquier M, Lim B B S, POP-TRAFFIC: A Novel Fuzzy Neural Approach to Road Traffic Analysis and Prediction, IEEE Transactions on Intelligent Transportation Systems, June 2006, pp. 133-146, DOI 10.1109/TITS.2006.874712.

15 Scherer H. R, Gotchev G, System WATERMAN for water monitoring and sustainable management, Proc. Summer School on Appl. Math. in Engineering, Sozopol 1999 (Chesankov B I, Todorov M D, eds.), Heron Press, Sofia, 2000, pp. 161-162.

16 Sugano M, Performance Analysis of Signaling Delay for Wireless Cellular Networks, Journal of Computing and Information Technology - CIT 12 (2004), no. 3, 251-262, DOI 10.2498/cit.2004.03.06.

17 Sun S, Zhang C, Yu G, A Bayesian Network Approach to Traffic Flow Forecasting, IEEE Transactions on Intelligent Transportation Systems, March 2006, pp. 124-132, DOI 10.1109/TITS.2006.869623.

18 Tang S, Gao H, Traffic-Incident Detection-Algorithm Based on Nonparametric Regression, IEEE Transactions on Intelligent Transportation Systems, March 2005, pp. 38-42, DOI 10.1109/TITS.2004.843112.

19 THEMIS project, 2004, available at http://themis-network.org/

20 TRACKSS project, 2006, available at http://www.trackss.net/

21 VIKING project, 2005, available at http://www.viking.ten-t.com

22 WATERMAN project, 2005, available at http://wWw.ima.ge.cnr.it/ waterman/ 